\title{
A Novel Multicasting Scheme over Wireless LAN Systems by Using Relay
}

\author{
Kang Jin Yoon, Tae Sung Kim, and Young Yong Kim \\ Department of Electrical and Electronic Engineering, Yonsei University, \\ 134 Shinchon-dong, Seodaemun-gu, Seoul, 120-749, Korea \\ \{mecem, xinia0214, y2k\}@yonsei.ac.kr
}

\begin{abstract}
We propose a novel multicast scheme that can provide quality-of-service (QoS) to multicast service over IEEE 802.11 wireless LANs by utilizing medium access control (MAC) layer relay. It is well known that IEEE 802.11 provides a physical layer multi-rate capability in response to different channel conditions, and hence packets may be delivered at a higher data rate through a relay node than through the direct link if the direct link has low quality and low data rate. We develop the distributed relay node selection algorithm and the relay channel selection algorithm. The effectiveness of proposed scheme is examined by numerical method and simulation. Simulations show that the proposed relayed multicast significantly improves throughput and delay performance.
\end{abstract}

\section{Introduction}

As wireless technologies grow rapidly, multimedia contents delivery through multicast scheme over the wireless networks is emerging as an important area in communication networks. We focus on multicast service over the Wireless Local Area Networks (WLANs) technology which is well known that IEEE 802.11 provides a physical layer multi-rate capability in response to different channel conditions. In the WLANs standard, IEEE 802.11b supports transmission rate of $1,2,5.5$, and $11 \mathrm{Mbps}$, and IEEE $802.11 \mathrm{~g}$ supports data rate of $1,2,5.5,6$, 9, 54 Mbps.

However, there is a problem that multicast data rate is limited by the node which has the lowest data rate. It can make more users received multicast data, but causes some nodes which are in good channel condition to lose their data rate. The degradation of multicast data rate can reduce total throughput, and then increase the drop ratio of the multimedia contents, which is due to an increment of the transmission delay. As a result, nodes may suffer poor quality-of-service (QoS).

Therefore, we propose a novel multicast scheme that can provide higher data rate for the QoS guarantee of the multicast service over IEEE 802.11 wireless LANs by utilizing MAC layer relay, called relayed multicast. In our proposed multicast scheme, the nodes which have a good channel relay multicast data to the bad channel nodes. For adopting relay scheme to the multicast, we design the distributed relay node selection algorithm which helps choosing the relay nodes 
among good channel nodes efficiently. We also develop the relay channel selection algorithm to pick a proper relay channel, which makes transmit simultaneously relayed data.

The rest of this paper is organized as follows. Section 2 gives an overview of the related works, as well as the motivation behind the design of our scheme. The details of relayed multicast including the distributed relay node selection and the relay channel selection algorithm are presented in Section 3. Section 4 describes the analysis of the relayed multicast and shows the simulation results that illustrate the effectiveness of the proposed schemes. We conclude the paper in section 5 .

\section{Related Works and Motivation}

In recent years, many papers proposed various solutions for the QoS guarantee of multimedia contents delivery over IEEE 802.11 [3, 4, 5]. Moreover, some researchers developed many relay schemes to acquire higher data rate and the QoS enhancement for the unicast service $[6,7,8]$. Nevertheless, to the best of our knowledge, there is no work have been studied for the QoS guarantee through the increment of the multicast transmission rate.

Data packets may be delivered at higher data rate through a relay node than through the direct link if the direct link has low quality and low data rate. Therefore the multi-rate capability can be further exploited by MAC layer multihop transmission. In [6], the authors propose the relay schemes to increase the unicast data rate in IEEE 802.11 networks. Similar to [6], by adopting a multihop relay into the multicast, we can acquire multicast data rate gain, which can guarantee better QoS for received nodes.

We consider a wireless network based on IEEE 802.11 WLANs operated as infrastructure mode. The physical layer uses IEEE 802.11b, and, similar to [9], we use multi-channel scheme that contains one general channel to communicate with $\mathrm{AP}$ and two relay channel to relay multicast data with single transceiver. To support MAC-level QoS for multicast data, the Enhanced Distributed Coordinate Function $(\mathrm{EDCF})$ is used for the MAC. The EDCF provides the differentiated channel access, which is achieved through varying the amount of time a station would sense the channel to be idle and the length of the contention window.

\section{Relayed Multicast}

To exploit the multi-rate capability of IEEE 802.11, we organize multicast nodes into two groups based on channel condition between the AP and a multicast node; the multicast group and the relayed multicast group. The nodes in the multicast group have a good channel condition enough to receive data packets at the $5.5 \mathrm{Mbps}$ data rate. The multicast group also includes all nodes which can receive data in $11 \mathrm{Mbps}$. The remaining nodes which are able to receive data packets at only $2 \mathrm{Mbps}$ are put into the relayed multicast group. 


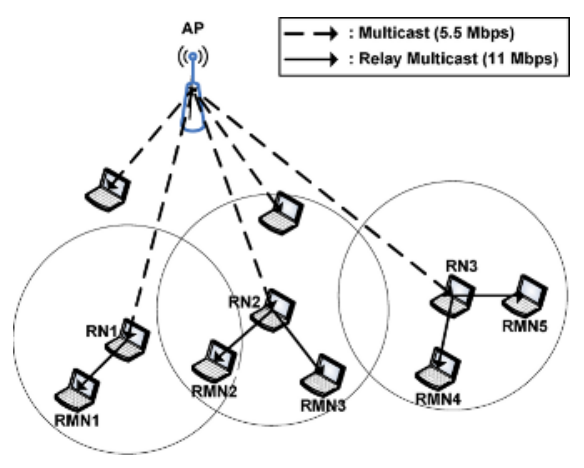

Fig. 1. An example of the relayed multicast

Fig. 1 illustrates an example of the relayed multicast. The Multicast group nodes (MNs) are connected with the AP by dotted lines, and the relayed multicast group members are connected with their relay nodes (RNs) by solid lines. First of all, the RMNs select their relay nodes out of the multicast group using the distributed relay node selection algorithm, and then choose relay channels to communicate with their relay nodes using the relay channel selection algorithm. Through these two methods, relayed multicast nodes (RMNs) can find the relay nodes appropriate for the relayed multicast that are able to relay data to the relayed multicast group in $11 \mathrm{Mbps}$.

\subsection{Distributed Relay Node Selection Algorithm}

In the wireless environments, because of the wireless multicast advantage [10], all MNs need not to relay multicast data. To relay efficiently, only a few nodes are needed. Moreover, the wireless multicast is the same mechanism as the broadcast in the physical layer. For reasons of that, it is not important for the RNs to know to transmit which RMNs. Instead of that, it is reasonable that RMNs decide to select their RNs. Our proposed selection algorithm, the distributed relay node selection, is designed in the relayed nodes side.

We assume receiver-initiated channel condition measurement and let the receiver notify the sender's transmission rate via control packets. Each node can overhear all ongoing control packets, and know the channel condition between the sender and itself by sensing the signal strength and extracting the piggybacked transmission rate in control packets.

At first, AP sends the multicast service advertisement (MSA) to whole nodes in its transmission range. The MNs respond to $\mathrm{AP}$ with the service request message (SRM). They put in their maximum data rate between AP and themselves in the SRM. The RMNs can overhear this message. By extracting the piggybacked transmission rate in the SRM, they find the nodes that are able to communicate with AP in 5.5 Mbps. Among those nodes, they select some nodes that have the signal strength of the SRM is high enough to communicate with 
themselves in $11 \mathrm{Mbps}$. Each $R M N_{i}$ has the individual the candidate set of the relay nodes, $X_{i}$.

After constructing $X_{i}, R M N_{i}$ sends the request to relay (RTR) message to the $M N$ which was sensed the SRM in the strongest signal strength. The node that receives the RTR will perform the relay nodes $\left(R N_{i}\right)$. At the same time, $R M N_{j}$ $(i \neq j)$, other nodes among the rest of the RMNs that are in the transmission rage of $R M N_{i}$ extract the RTR, and then check the destination address. If the address is the entry of their candidate set $X_{j}$, they also set that node as their RN. They do not have to send RTR to the other MNs. The algorithm continues until all RMNs have their RNs.

\subsection{Relay Channel Selection Algorithm}

In conventional IEEE 802.11 WLANs that operate under single channel PHY, all nodes in the transmission range of transmitting node should wait until the end of transmission because it protects collisions. However, it leads to transmission delay when the RNs relay data. In that case, the relayed multicast may show lower performance than conventional multicast.

To solve this problem, we utilize the multi-channel approach. As we mentioned section 2, there are 3 non-overlap channels in the IEEE 802.11. Unfortunately, with one receiver constraint, the standard only defines the MAC operations for single channel mode. We design a simple multi-channel MAC algorithm for relayed multicast. We use 3 channels $\left(C_{1}, C_{2}\right.$, and $\left.C_{3}\right)$ for relay, and the algorithm operates as below.

At first, $R N_{i}$ broadcasts the channel assignment message (CAM) for $C_{1}$. After receiving CAM from $R N_{i}, R M N_{i j}$ which is in the overlap zone checks the relay channel list (RCH). If $C_{1}$ is not in the list, $C_{1}$ is entered into the $\mathrm{RCH}=C_{1}$, and $R M N_{i j}$ send the channel assignment success message (CAS) to $R N_{i}$.

Then, $R N_{j}$ broadcasts the CAM for $C_{1}$. At this time, because $C_{1}$ is the entry of RCH, RMNij send the channel assignment failure message (CAF) to $R N_{j}$. In the $\mathrm{CAF}$, the available relay channel is piggybacked. In this case $C_{2}$ is available, so $R N_{j}$ broadcasts the CAM for $C_{2}$. After receiving CAM for $C_{2}$ from $R N_{j}$, $R M N_{i j}$ enters $C_{2}$ into the $\mathrm{RCH}=C_{1}, C_{2}$, and then sends the CAS to $R N_{j}$.

\subsection{Temporal Operation of the Relayed Multicast}

We now consider the temporal operation of the relayed multicast. Transmission/reception activity between AP and nodes is used a general channel (G-channel). In addition, each $\mathrm{RN}$ has a relay channel (R-channel) it uses to relay multicast data from AP to RMNs. Because we assume a single transceiver case, each node has only a single channel at a time. Thus, we use a new frame for changing channel before relay operation, the relay channel change message ( $\mathrm{RCH})$. If RMNs receive the $\mathrm{RCH}$ from their RNs, they jump to their R-channels that were determined by using the relay channel selection algorithm. After receiving relayed data, RMNs return to the G-channel. For a reliable MAC-layer multicast, we adopt leader-based-ack mechanism. A leader of each group is selected by the first node to send the SRM and RTR. 
Table 1. Simulation Parameters

\begin{tabular}{|c|c||c|c|}
\hline Paylod & 8000 bits & SIFS & $10 \mu \mathrm{s}$ \\
\hline MAC header & 224 bits & AIFS $(3)$ & SIFS $+1^{*}$ Slot time \\
\hline PHY header & $192 \mathrm{bits}$ & AIFS $(2)$ & SIFS $+1^{*}$ Slot time \\
\hline ACK, CTS & 112 bits + PHY header & AIFS $(1)$ & SIFS $+1^{*}$ Slot time \\
\hline RTS, CCM & 160 bits + PHY header & AIFS $(0)$ & SIFS $+1^{*}$ Slot time \\
\hline Slot time & $20 \mu \mathrm{s}$ & CWmin $(0 \ldots 3)$ & {$[31,31,15,7]$} \\
\hline Simulation time & $100 \mathrm{~s}$ & CWmax $(0 \ldots 3)$ & {$[1023,1023,31,15]$} \\
\hline
\end{tabular}

Our proposed scheme operates in the following order.

1. The AP multicasts data to the multicast group in the $5.5 \mathrm{Mbps}$ after waiting $\operatorname{AIFS}(1)$ and backoff when the channel is sensed idle.

2. A leader of the multicast group sends an ACK to the AP after SIFS.

3. RNs send the RCH to the RMNs, and then relay the data after SIFS.

4. Leaders of RMNs respond to RNs with the ACK, while other RMNs return to the RMNS by overhearing the ACK.

\section{Performance Evaluation}

In this section, the performances of our design is examined by the numerical method and our event-driven simulation program, written in the MATLAB. In order to select a suitable data rate, we assume the distance thresholds for $11 \mathrm{Mbps}, 5.5 \mathrm{Mbps}$, and $2 \mathrm{Mbps}$ are $100 \mathrm{~m}, 200 \mathrm{~m}$, and $250 \mathrm{~m}$ respectively, similar to [12]. The payload size for best effort is set to be 1000 bytes and all nodes are always backlogged. The best effort data uses $\mathrm{AC}(0)$, and RTS/CTS mechanism. To support QoS for multicast data and relay data, $\mathrm{AC}(1)$ and $\mathrm{AC}(2)$ are adopted respectively. Nodes are uniformly distributed centering around AP, and the service area is within $250 \mathrm{~m}$ radius of the AP. Other simulation and IEEE $802.11 \mathrm{e}$ MAC parameters are set as in Table 1 . We investigate the overall throughput and transmission delay compared with conventional multicast service which uses only $2 \mathrm{Mbps}$. With this setup, we investigate the overall throughput, transmission delay performances.

\subsection{Numerical Result}

In [7], Hao Zhu and Guohong Cao show an analysis of their proposed relay scheme for unicast traffic by using Bianchi's Markov Chain model [11]. In this section, similar to [7], we analyze the saturation throughput gain of the relayed multicast as compared with conventional multicast operating 2 Mbps.

For simplicity, we assume the channel condition is ideal, and do not apply IEEE 802.11e in this analysis. Assume that each node applies the binary 
exponential backoff algorithm with the maximum backoff stage $m$, the number of flows is represented $n$, and the initial backoff window size $W$. Then, the probability $\tau$ that a flow transmits in a slot time is obtained from the following functions:

$$
\begin{gathered}
\tau=\frac{2(1-2 p)}{(1-2 p)(W+1)+p W\left(1-(2 p)^{m}\right)} \\
p=1-(1-\tau)^{n-1}
\end{gathered}
$$

$p$ denotes the conditional collision probability that a transmitted packet encounters a collision because at least one of the $n-1$ remaining nodes transmit in the same time slot.

If there are no hidden terminals, multicast is exactly same as unicast operation because we can treat the set of receiver as one node. In the relayed multicast case, if it is possible for RNs to transmit concurrently to their RMNs, the relayed multicast can be analyzed similar to multicast. There exists only one difference that relayed multicast uses DCF scheme twice; for the multicast, and the relay.

$T s^{M}, T s_{M}^{R M}$, and $T s_{R}^{R M}$ are denoted the average time for the channel being sensed busy because of successful transmission under conventional multicast, multicast from AP to MNs, relay from RNs to RMNs. $T c^{M}, T c_{M}^{R M}$, and $T c_{R}^{R M}$ are the average time for the channel being sensed busy because of during a collision conventional multicast, multicast from AP to MNs, relay from RNs to RMNs respectively:

$$
\begin{aligned}
T s^{M}=D I F S+\operatorname{data}(2 M b p s)+S I F S+A C K+2 \times \delta \\
T c^{M}=D I F S+\operatorname{data}(2 M b p s)+\delta \\
T s_{M}^{R M}=D I F S+\operatorname{data}(5.5 M b p s)+S I F S+A C K+2 \times \delta \\
T c_{M}^{R M}=D I F S+\operatorname{data}(5.5 M b p s)+\delta \\
T s_{R}^{R M}=D I F S+R C H+\text { SIFS }+\operatorname{data}(11 M b p s)+\text { SIFS } \\
\quad+A C K+3 \times \delta \\
T c_{R}^{R M}=D I F S+R C H+\text { SIFS }+\operatorname{data}(11 M b p s)+2 \times \delta
\end{aligned}
$$

The data includes the overhead of PHY and MAC header and payload, and $\delta$ means propagation delay. From the result of [11], the average time to transmit one packet is calculated as follows. $T_{M}$ and $T_{R M}$ correspond the average packet transmission time under conventional multicast and relayed multicast:

$$
\begin{aligned}
T_{M}= & \left(1-P_{t r}\right) \sigma+P_{t r} P_{s} T s^{M}+P_{t r}\left(1-P_{s}\right) T c^{M} \\
T_{R M} & =\left(1-P_{t r}\right) \sigma+P_{t r} P_{s} T s_{M}^{R M}+P_{t r}\left(1-P_{s}\right) T c_{M}^{R M} \\
& +\left(1-P_{t r}\right) \sigma+P_{t r} P_{s} T s_{R}^{R M}+P_{t r}\left(1-P_{s}\right) T c_{R}^{R M}
\end{aligned}
$$


$\sigma$ is the duration of an empty slot time. $P_{t r}$ is the probability that there is at least one transmission in the considered slot time, and $P_{s}$ means the probability that a transmission occurring on the channel is successful.

$$
\begin{gathered}
P_{t r}=1-(1-\tau)^{n} \\
P_{s}=\frac{n \tau(1-\tau)^{n-1}}{P_{t r}}=\frac{n \tau(1-\tau)^{n-1}}{1-(1-\tau)^{n}}
\end{gathered}
$$

Then, the ratio between the saturation throughput of conventional multicast and relayed multicast is represented in the proportion of $T_{M}$ to $T_{M}$, denoted by $T_{M}$.

$$
\begin{aligned}
\gamma & =\frac{T_{M}}{T_{R M}} \quad\left(1-P_{t r}\right) \sigma+P_{t r} P_{s} T s^{M}+P_{t r}\left(1-P_{s}\right) T c^{M} \\
& =\frac{c_{t r}^{R}}{2\left(1-P_{t r}\right) \sigma+P_{t r} P_{s}\left(T s_{M}^{R M}+T s_{R}^{R M}\right)+P_{t r}\left(1-P_{s}\right)\left(T c_{M}^{R M}+T s_{R}^{R M}\right)}
\end{aligned}
$$

We show the numerical analysis of the saturation throughput gain as the function of payload size, and also validate our analysis through simulation. We assume $m=5, n=5$, and $W=31$. As shown in Fig. 2, the gap between numerical result and simulation is slightly small. This gap is due to transmit an RCH before relaying. We assumed every RN can transmit simultaneously, but they have different start times since each RN contends to get a transmission opportunity of RCH in the G-channel. We can see that throughput gain increases as payload size increases. There are additional overheads in relayed multicast because of transmitting RCH and relaying data packet. However, since overheads are much smaller than the reduced transmission time by relaying, relayed multicast always shows better performance than conventional multicast regardless of payload size.

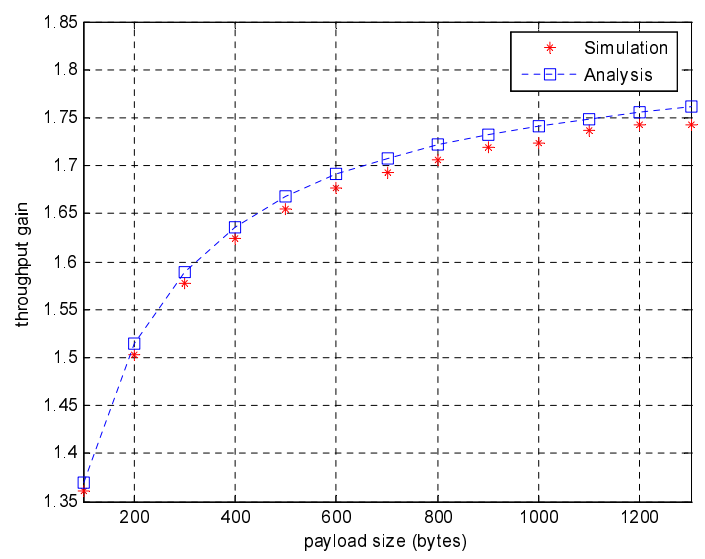

Fig. 2. Throughput gain of relayed multicast 


\subsection{Simulation Results}

Throughput Enhancement. Fig. 3 shows the system throughput of relayed multicast and conventional multicast. Throughput is calculated by the total amount of payload (in bits) delivered divided by the simulation time. The maximum throughput gain, $28 \%$, can be achieved in the case that user density is 0.3 . Throughput curve decrease exponentially according to increase user density, and when the user density is over 7 , the throughput of relayed multicast is less than that of conventional multicast. The reason of throughput decrease is MAC protocol of IEEE 802.11 which use contention based CSMA/CA. Relayed multicast needs 2 contention period, one is for multicast to MNs and the other is for relay to RMNs. Thus, the collision and waiting time will increase as the user density increase. In relayed multicast, since RCH message is transmitted in G-channel, it causes the additional transmission delay at the RNs, and then the efficiency of relayed multicast decrease rapidly.

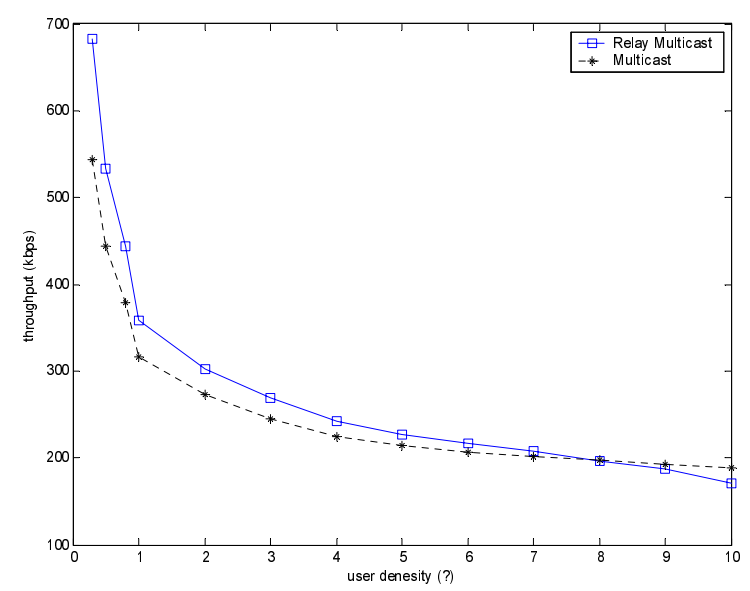

Fig. 3. The throughput comparison

Transmission Delay Performances. Fig. 4 and 5 show the transmission delay performances according to user density and data rate. Each case use data rate $=256 \mathrm{Kbps}$ and user density $=1$. Both cases, relayed multicast shows much better performance. Especially, when user density is fixed, relayed multicast keeps transmission delay under 1 second. Through these results, despite additional transmission for relay packets, relayed multicast can reduce transmission delay compared with conventional multicast. Considering that almost multicast are video services, relayed multicast needs less buffering time than that of conventional multicast to maintain the video quality. 


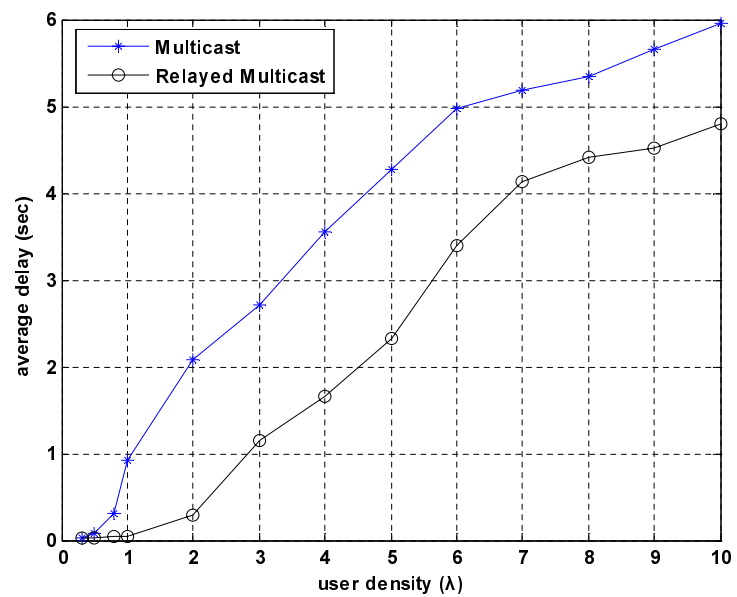

Fig. 4. The transmission delay according to user density

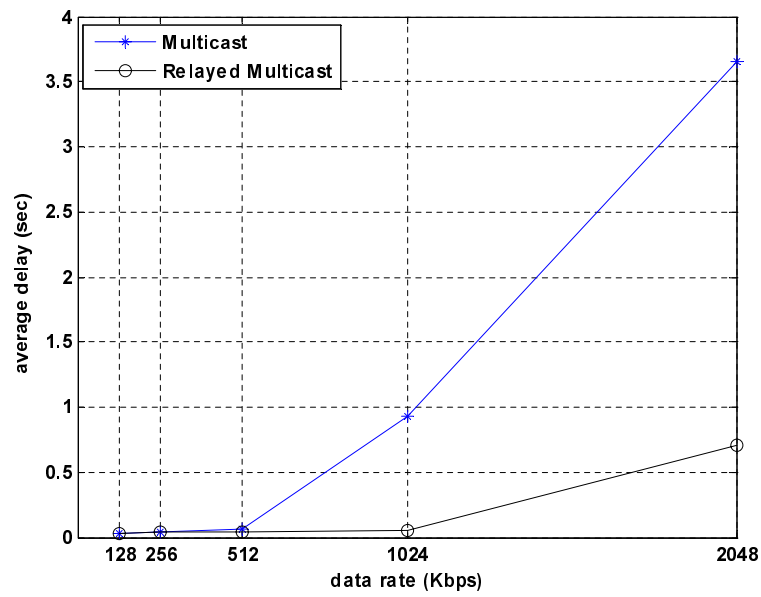

Fig. 5. The transmission delay according to data rate

\section{Conclusions}

In this paper, we design a new multicast scheme for IEEE 802.11 wireless LANs by utilizing MAC layer relay, called relayed multicast. For effective relayed multicast, we develop the distributed relay node selection and relay channel selection algorithm. Numerical results and Simulation show that the proposed scheme can increase the throughput, and significantly reduce the transmission delay. Therefore, multicast nodes can be served higher data rate multicast service and guaranteed the QoS of the multimedia service. 


\section{Acknowledgement}

This research was supported by the MIC(Ministry of Information and Communication), Korea, under the ITRC(Information Technology Research Center) support program supervised by the IITA(Institute of Information Technology Assessment)(IITA-2005-C1090-0502-0012).

\section{References}

1. IEEE 802.11 WG, Part 11: Wireless LAN Medium Access Control (MAC) and Physical Layer (PHY) specifications, ANSI/IEEE Std 802.11, 1999 Edition.

2. IEEE 802.11b WG, Part 11: Wireless LAN Medium Access Control (MAC) and Physical Layer (PHY) specifications, IEEE Std 802.11b-1999/Cor 1-2001, November 2001.

3. Miheala van der Schaar, Santhana Krishnamachari, Sunghyun Choi, and Xiaofeng $\mathrm{Xu}$, : Adaptive Cross-Layer Protection Strategies for Robust Scalable Video Transmission Over 802.11 WLANs, in IEEE J. Select. Areas Commun., vol. 21, NO. 10, December 2003.

4. Qiong Li, and Mihaela van der Schaar,: Providing Adaptive QoS to Layered Video Over Wireless Local Area Networks Through Real-Time Retry Limit Adaptation, IEEE Transaction on Multimedia, vol. 6, NO. 2, April 2004.

5. Abhik Majumdar, Daniel Grobe Sachs, Igor V. Kozintsev, and Minerva M. Yeung, Multicast and Unicast Real-Time Video Streaming Over Wireless LANs, IEEE Trans. on Circuits and Systems for Video Technology, vol. 12, NO. 6, June 2002.

6. Hao Zhu, and Guohong Cao,: On Improving the Performance of IEEE 802.11 with Relay-Enabled PCF, ACM/Kluwer Mobile Networks and App. 9, 423-434, 2004.

7. Hao Zhu, and Guohong Cao,: rDCF: A Relay-Enabled Medium Access Control Protocol for Wireless Ad Hoc Networks, IEEE INFOCOM 2005.

8. Dongmei Zhao, and Terence D. Todd,: Real-Time Traffic Support in Relayed Wireless Access Networkd Using IEEE 802.11, IEEE Wireless Commun., April 2004.

9. Jenhui Chen, Shiann-Tsong Sheu, and Chin-An Yang,: A New Multichannel Access Protocol for IEEE 802.11 Ad Hoc Wireless LANs, IEEE PIMRC 2003.

10. J. Wieselthier, G. Nguyen, and A. Ephremides,: On the construction of energyefficient broadcast and multicast trees in wireless networks, IEEE INFOCOM 2000.

11. G. Bianchi,: Performance Analysis of the IEEE 802.11 Distributed Coordination Function, IEEE J. Select. Areas Commun., pp. 535-547, March 2000.

12. B. Sadeghi, V. Kanodia, A. Sabharwal and E. Knightly,: Opportunistic media access for multirate Ad Hoc networks, ACM Mobicom 2002, July 2001. 